University of Nebraska - Lincoln

DigitalCommons@University of Nebraska - Lincoln

1998

\title{
The master equation approach to configurational kinetics of alloys via the vacancy exchange mechanism: general relations and features of microstructural evolution
}

Kirill D. Belashchenko

University of Nebraska-Lincoln, belashchenko@unl.edu

V G Vaks

'Kurchatov Institute' Russian Research Centre, vaks@mbslab.kiae.ru

Follow this and additional works at: https://digitalcommons.unl.edu/physicsbelashchenko

Belashchenko, Kirill D. and Vaks, V G, "The master equation approach to configurational kinetics of alloys via the vacancy exchange mechanism: general relations and features of microstructural evolution" (1998). Kirill Belashchenko Publications. 2.

https://digitalcommons.unl.edu/physicsbelashchenko/2

This Article is brought to you for free and open access by the Research Papers in Physics and Astronomy at DigitalCommons@University of Nebraska - Lincoln. It has been accepted for inclusion in Kirill Belashchenko Publications by an authorized administrator of DigitalCommons@University of Nebraska - Lincoln. 


\title{
The master equation approach to configurational kinetics of alloys via the vacancy exchange mechanism: general relations and features of microstructural evolution
}

\author{
K D Belashchenko and V G Vaks \\ Russian Research Centre 'Kurchatov Institute', Moscow 123182, Russia
}

Received 27 October 1997

\begin{abstract}
The earlier-suggested generalized Gibbs distribution approach to the configurational kinetics of non-equilibrium alloys is extended to the case of many-component alloys and the realistic vacancy-mediated atomic exchange mechanism is incorporated. Exact and approximate equations for the temporal evolution of atomic distributions as well as for the free energy of a non-equilibrium alloy are presented. It is shown that the evolution of the main alloy component distribution for the nearest-neighbour vacancy exchange model can usually be described in terms of an equivalent direct exchange model. This conclusion is illustrated with the computer simulation of decomposition and ordering via the vacancy exchange mechanism for a twodimensional alloy model. The simulation also reveals the localized ordering phenomenon at very early stages of ordering, in agreement with previous experiments and Monte Carlo simulation, but with no interfacial vacancy trapping which was suggested in previous works in order to explain this phenomenon.
\end{abstract}

\section{Introduction}

The problem of possible influence of the atomic exchange mechanism on the configurational kinetics and microstructural evolution of alloys recently received some attention [1-9]. In most of the theoretical treatments of these problems (see e.g. [10-15]) the simplified direct exchange model was used. This model assumes direct exchange of positions between unlike neighbouring atoms in an alloy, while actually the exchange occurs between the main alloy component atoms (e.g. A or B atoms in an A-B alloy) and the neighbouring vacancies. As the vacancy concentration $c_{\mathrm{v}}$ in alloys is actually quite small, $c_{\mathrm{v}} \lesssim 10^{-4}$, employing the direct exchange model greatly simplifies the theoretical studies of microstructural evolution by reducing the computation times by several orders of magnitude. However, it is not clear a priori whether using the unrealistic direct exchange model results in some errors or missing some effects in the theoretical description. In particular, a notable segregation of vacancies at interphase or antiphase boundaries was observed in theoretical studies of some alloy models $[1-5,7-9,16]$, and the problem of possible influence of this segregation on the microstructural evolution was discussed by a number of authors.

Most of these studies used Monte Carlo simulation and found the main features of the evolution via the direct and vacancy exchange mechanisms to be similar. In particular, the asymptotic growth law for the mean size of precipitates under spinodal decomposition, $R_{p} \sim t^{1 / 3}$, and that of the ordered domain size under alloy ordering, $R_{d} \sim t^{1 / 2}$, were usually observed for both kinetic mechanisms [1-3,6,7], except in [4] and [5] where some 
differences for the asymptotic ordered domain growth law have been found. Athenes et al [7] also observed some peculiar features of the vacancy-mediated kinetics at very early stages of ordering which are discussed below in section 5. Chen and Geng $[8,9]$ simulated the vacancy-mediated spinodal decomposition and ordering employing some kinetic equations [18] that seem to be not entirely consistent [15]. The results of [8] and [9] notably differ from those of other works $[1,4,16]$, in particular in the degree of vacancy segregation at interphase and antiphase boundaries, which seems to be one more indication of unreliability of equations proposed in [18].

In the present paper we use the analytical approach to the description of the alloy configurational kinetics based on the fundamental master equation for probabilities of various distributions of atoms over lattice sites. The main ideas of this approach have been discussed in [14] and [15] using the direct atomic exchange model for a binary alloy AB. As actually the exchange is realized via vacancies, to realistically describe the kinetics one should consider many-component alloys with at least three species, such as $\mathrm{ABv}$ alloys. In a recent paper [16] we studied stationary distributions of vacancies in an $\mathrm{ABv}$ alloy and showed that they often tend to segregate at interphase or antiphase boundaries. In the present work we extend this approach to the dynamical problems.

In section 2 we generalize the master equation approach described in [15] to the manycomponent alloy case. We derive, in particular, the exact kinetic equations for local concentrations and for the free energy of a nonequilibrium alloy. We show that the free energy has the fundamental property of not increasing in the spontaneous evolution of the system, similarly to the Boltzmann's non-decreasing entropy. In section 3 we present the basic kinetic equation in the cluster field approximation. We also discuss the expressions for generalized driving forces and mobilities in this equation using the mean-field and the pair cluster approximations. The results of sections 2 and 3 are used in section 4 to study some general features of the vacancy-mediated kinetics. We show that for the conventional nearest-neighbour vacancy exchange model the kinetics can usually be described in terms of an equivalent direct exchange model with certain effective direct exchange rates. In section 5 we present the results of our computer simulation of vacancy-mediated decomposition and ordering for a 2D alloy model which illustrate the conclusions of section 4 . For very early stages of ordering the simulation also reveals the localized ordering phenomenon that was discussed earlier in [19] and [7], but our results seem to suggest new features in the interpretation of this phenomenon. Our main results are summarized in section 6 .

\section{Exact equations for averages of occupation numbers and for free energy of a many-component non-equilibrium alloy}

In this section we generalize the master equation approach described in [15] to the manycomponent alloy case. Our arguments follow those of [15] for a binary alloy with necessary generalizations. We consider the substitutional alloy that includes atoms of $m$ different species $\mathrm{p}=\mathrm{p}_{1}, \mathrm{p}_{2}, \ldots, \mathrm{p}_{m}$, in particular vacancies for which $\mathrm{p}=\mathrm{v}$. Various distributions of atoms over lattice sites $i$ are described with the different occupation number sets $\left\{n_{\mathrm{p} i}\right\}$ where the operator $n_{\mathrm{p} i}$ is unity when the site $i$ is occupied by a p-species atom and zero otherwise. For each $i$ these operators obey the identity $\sum_{\mathrm{p}} n_{\mathrm{p} i}=1$, so only $m-1$ of them are independent. It is convenient to mark the independent operators with special symbols, e.g. with greek letters: $\left(n_{\mathrm{p} i}\right)_{\text {indep }}=n_{\alpha i}$, while the rest operator denoted as $n_{\mathrm{r} i}$ is expressed via $n_{\alpha i}$ :

$$
n_{\mathrm{r} i}=1-\sum_{\alpha} n_{\alpha i} .
$$


In calculations it is usually convenient to put ' $r$ ' in (1) to be one of the main components, e.g. $\mathrm{r}=\mathrm{B}$ in the $\mathrm{ABv}$ alloy, as then the resulting equations explicitly include the vacancy operators $n_{\mathrm{v} i}$ being on average small: $\left\langle n_{\mathrm{v} i}\right\rangle=c_{\mathrm{v} i} \ll 1$. However, in general discussions, such as the 'H-theorem' below, it may be convenient to eliminate the vacancy operators by putting $\mathrm{r}=\mathrm{v}$ in (1).

In terms of all operators $n_{\mathrm{p} i}$ the configurational Hamiltonian $H^{t}$ can be written as

$$
H^{t}=\sum_{\mathrm{p}} \Phi_{i}^{\mathrm{p}} n_{\mathrm{p} i}+\frac{1}{2} \sum_{\mathrm{pq}, i j} V_{i j}^{\mathrm{pq}} n_{\mathrm{p} i} n_{\mathrm{q} j}+\frac{1}{3 !} \sum_{\mathrm{pqr}, i j k} V_{i j k}^{\mathrm{pqr}} n_{\mathrm{p} i} n_{\mathrm{q} j} n_{\mathrm{r} k}+\cdots
$$

Here $\Phi_{i}^{\mathrm{p}}$ are possible external fields (which are present only if not all alloy sites are equivalent), and $V_{i \ldots j}^{\mathrm{p} \ldots \mathrm{q}}$ are the interaction potentials. After elimination of the operators $n_{\mathrm{ri}}$ according to (1), i.e in terms of only independent $n_{\alpha i}$, the Hamiltonian takes the form

$H=E_{0}+\sum_{\alpha i} \varphi_{i}^{\alpha} n_{\alpha i}+H_{i n t}=E_{0}+\sum_{\alpha i} \varphi_{i}^{\alpha} n_{\alpha i}+\sum_{\alpha \beta, i>j} v_{i j}^{\alpha \beta} n_{\alpha i} n_{\beta j}+\cdots$

where $E_{0}, \varphi_{i}^{\alpha}$ and $v_{i \ldots j}^{\alpha \ldots \beta}$ are linearly expressed via $\Phi_{i}^{\mathrm{p}}$ and $V_{i \ldots j}^{\mathrm{p} \ldots \mathrm{q}}$ in (2). In particular, for the effective fields $\varphi_{i}^{\alpha}$ and pair interactions $v_{i j}^{\alpha \beta}$ we have

$\varphi_{i}^{\alpha}=\left(\Phi_{i}^{\alpha}-\Phi_{i}^{\mathrm{r}}\right)+\sum_{j}\left(V^{\alpha \mathrm{r}}-V^{\mathrm{rr}}\right)_{i j}+\sum_{j>k}\left(V^{\alpha r r}-V^{\mathrm{rrr}}\right)_{i j k}+\cdots$

$v_{i j}^{\alpha \beta}=\left(V^{\alpha \beta}-V^{\alpha \mathrm{r}}-V^{\mathrm{r} \beta}+V^{\mathrm{rr}}\right)_{i j}+\sum_{k}\left(V^{\alpha \beta \mathrm{r}}-V^{\alpha \mathrm{rr}}-V^{\mathrm{r} \beta \mathrm{r}}+V^{\mathrm{rrr}}\right)_{i j k}+\cdots$.

The fundamental master equation for the probability $P$ of finding the occupation number set $\left\{n_{\alpha i}\right\}=\xi$ is

$$
\mathrm{d} P(\xi) / \mathrm{d} t=\sum_{\eta}[W(\xi, \eta) P(\eta)-W(\eta, \xi) P(\xi)] \equiv \hat{S} P
$$

where $W(\xi, \eta)$ is the $\eta \rightarrow \xi$ transition probability.

Adopting the 'thermally activated atomic exchange model' $[11,13]$ for probabilities $W$ in (5), we can express the transfer matrix $\hat{S}$ in (5) in terms of the probability $W_{i j}^{\mathrm{pq}}$ of an elementary inter-site exchange ('jump') q $j \leftrightarrow \mathrm{p} i$ (see [13] for details):

$$
W_{i j}^{\mathrm{pq}}=n_{\mathrm{p} i} n_{\mathrm{q} j} \omega_{i j}^{\mathrm{pq}} \exp \left[-\beta\left(E_{\mathrm{p} i, \mathrm{q} j}^{s}-\hat{E}_{\mathrm{p} i, \mathrm{q} j}^{i n}\right)\right] \equiv n_{\mathrm{p} i} n_{\mathrm{q} j} \gamma_{i j}^{\mathrm{pq}} \exp \left(\beta \hat{E}_{\mathrm{p} i, \mathrm{q} j}^{i n}\right) .
$$

Here $\omega_{i j}^{\mathrm{pq}}$ is the attempt frequency, $\beta=1 / T$ is the reciprocal temperature, $E_{\mathrm{p} i, \mathrm{q} j}^{s}$ is the saddle point energy, $\gamma_{i j}^{\mathrm{pq}}$ is the configurationally independent factor in the jump probability, and $\hat{E}_{\mathrm{p} i, \mathrm{q} j}^{i n}$ is the initial (before the jump) configurational energy of jumping atoms. If we accept for simplicity the pair interaction model, i.e. retain only the first two terms in the Hamiltonian (2), then the operator $\hat{E}_{\mathrm{p} i \mathrm{q} j}^{i n}$ in equation (5) may be expressed in terms of formal variational derivatives of the Hamiltonian (2) over $n_{\mathrm{p} i}$ and $n_{\mathrm{q} i}, H_{\mathrm{p} i}^{t}=\delta H^{t} / \delta n_{\mathrm{p} i}$ and $H_{\mathrm{p} i, \mathrm{q} j}^{t}=\delta^{2} H^{t} / \delta n_{\mathrm{p} i} \delta n_{\mathrm{q} j}$ :

$$
\hat{E}_{\mathrm{p} i, \mathrm{q} j}^{i n}=n_{\mathrm{p} i} H_{\mathrm{p} i}^{t}+n_{\mathrm{q} j} H_{\mathrm{q} j}^{t}-n_{\mathrm{p} i} n_{\mathrm{q} j} H_{\mathrm{p} i, \mathrm{q} j}^{t}
$$

where the third term corresponds to the subtraction of the 'double-counted' interaction between atoms $\mathrm{p}$ and $\mathrm{q}$ at sites $i$ and $j$. The operators $n_{\mathrm{r} i}$ in (7) and below have been expressed via the independent $n_{\alpha i}$ according to (1). When many-particle, non-pairwise interactions are present, the simple kinetic model (5), (6) may need some refinement to ensure the detailed balance principle (for example, if many-particle correlations are taken into account in the treatment used). 
Multiplying (5) by operators $n_{\alpha i}, n_{\alpha i} n_{\beta j}$ etc, and summing over all configurational states, i.e. over all number sets $\left\{n_{\alpha i}\right\}$, we obtain the set of equations for averages $\left\langle n_{\alpha i} n_{\beta j} \ldots n_{\gamma k}\right\rangle \equiv g_{i j \ldots k}^{\alpha \beta \ldots \gamma}$, in particular, for the local concentration $c_{\alpha i}=\left\langle n_{\alpha i}\right\rangle=g_{i}^{\alpha}$ :

$$
\mathrm{d} g_{i j \ldots k}^{\alpha \beta \ldots \gamma} / \mathrm{d} t=\left\langle n_{\alpha i} n_{\beta j} \ldots n_{\gamma k} \hat{S}\right\rangle
$$

where $\langle(\ldots)\rangle=\operatorname{Tr}\{(\ldots) P\}$ means the averaging over the distribution $P$, i.e. the summation of the operator product $(\ldots) P$ over all occupation number sets $\{\xi\}$.

Using (5) and (6) we can write (8) as $[13,15]$

$$
\begin{aligned}
& \frac{\mathrm{d}}{\mathrm{d} t} g_{i j \ldots k}^{\alpha \beta \ldots \gamma}=\sum_{\mathrm{p} ; s \neq i \neq j \neq \ldots k}\left\langle\left(\gamma_{i s}^{\mathrm{p} \alpha} n_{\mathrm{p} i} n_{\alpha s} \mathrm{e}^{\beta E_{\mathrm{p}, \alpha s}^{i n}}-\gamma_{s i}^{\mathrm{p} \alpha} n_{\mathrm{p} s} n_{\alpha i} \mathrm{e}^{\beta E_{\mathrm{p}, \alpha i}^{i n}}\right) n_{\beta j} \ldots n_{\gamma k}\right\rangle \\
& +\{\alpha i \rightarrow \beta j, \ldots \gamma k\}
\end{aligned}
$$

where $\{\alpha i \rightarrow \beta j, \ldots \gamma k\}$ denotes the sum of expressions obtained from the first term in (9) by index permutation.

Since $n_{\alpha i}$ are the projection operators $n_{\alpha i} n_{\beta i}=\delta_{\alpha \beta} n_{\alpha i}$, the most general expression for the distribution function $P(\xi)=P\left\{n_{\alpha i}\right\}$ in (5) can be written as

$$
P\left\{n_{\alpha i}\right\}=\exp \left[\beta\left(\Omega+\sum_{\alpha i} \lambda_{i}^{\alpha} n_{\alpha i}-Q\right)\right] \equiv \exp \left[\beta\left(\Omega-Q^{\prime}\right)\right] .
$$

Here the 'quasi-Hamiltonian' $Q^{\prime}$ is

$$
Q^{\prime}=-\sum_{\alpha i} \lambda_{i}^{\alpha} n_{\alpha i}+Q=-\sum_{\alpha i} \lambda_{i}^{\alpha} n_{\alpha i}+\sum_{\alpha \beta, i>j} a_{i j}^{\alpha \beta} n_{\alpha i} n_{\beta j}+\cdots
$$

the 'quasi-interaction' term $Q$ is an analogue of the interaction $H_{\text {int }}$ in (3), and the generalized grand canonical potential $\Omega$ is determined by the normalizing condition:

$$
\Omega=-T \ln \operatorname{Tr} \exp \left(-\beta Q^{\prime}\right) \text {. }
$$

For what follows it is convenient to rewrite (9) in a form more symmetrical with respect to the permutation of indices $i$ and $s$. To this end we make manipulations analogous to those used in [15]. We perform summation over all possible occupations of sites $i$ and $s$, i.e. over all values of $n_{\delta i}$ and $n_{\epsilon s}$ (being either zero or unity) in the first term of (9). Due to the presence of the projection operator $n_{\mathrm{p} i} n_{\alpha s}$ in this term, the summation reduces to putting $n_{\mathrm{q} i}=\delta_{\mathrm{qp}}$ and $n_{\epsilon s}=\delta_{\epsilon \alpha}$ in the $n_{\mathrm{q} i}$ - and $n_{\epsilon s}$-dependent exponential factor $\exp Y$ multiplied by this projection operator, where

$$
Y=\beta\left(E_{\mathrm{p} i, \alpha s}^{i n}-Q^{\prime}\right) .
$$

To employ the well elaborated calculation methods of statistical physics to the obtained relation it is convenient to restore formally the summation over all occupation number sets $\{\xi\}$ in (9), including all values of $n_{\delta i}$ and $n_{\epsilon s}$. To this end we can introduce the operator $n_{\mathrm{ri} i} n_{\mathrm{rs}}$ in the summand. Since this projection operator is nonzero only when all $n_{\delta i}$ and $n_{\epsilon s}$ are zero, the summation with this factor over all possible occupations of sites $i$ and $s$ is equivalent to omitting all $n_{\delta i}$ - and $n_{\epsilon s}$-dependent terms in the exponential $\exp Y$. Therefore, the result of the summation can be written as

$$
\sum_{n_{\delta i}, n_{\epsilon s}} n_{\mathrm{p} i} n_{\alpha s} \exp Y=\sum_{n_{\delta i}, n_{\epsilon s}} n_{\mathrm{ri}} n_{\mathrm{r} s} \exp \left(Y_{\mathrm{p} i}+Y_{\alpha s}+Y_{\mathrm{p} i, \alpha s}+Y\right) .
$$

Here $Y_{\mathrm{p} i}, Y_{\alpha s}$ and $Y_{\mathrm{p} i, \alpha s}$ are the variational derivatives of the operator $Y$ over the relevant occupation numbers: $Y_{\mathrm{p} i}=\delta Y / \delta n_{\mathrm{p} i}$, etc. The first, second or third term of the exponential in (13) corresponds to the contribution to the sum (13) of the term in $Y$ linear in $n_{\mathrm{p} i}$ but not in $n_{\alpha s}$, linear in $n_{\alpha s}$ but not in $n_{\mathrm{p} i}$ or linear in both $n_{\mathrm{p} i}$ and $n_{\alpha s}$, respectively. At $\mathrm{p}=\mathrm{r}$ 
the derivatives $Y_{\mathrm{p} i}$ and $Y_{\mathrm{p} i, \alpha s}$ in (13) should be put zero as the operator $Y$ in (13) depends only on the independent variables $n_{\delta i}$ but not on $n_{\mathrm{r} i}$ (this rule can be checked directly by putting $\mathrm{p}=\mathrm{r}$ in (9)).

Analogous manipulations in the second term of (9) correspond just to the interchange of indices $i \leftrightarrow s$. Finding the variational derivatives in (13) with the use of (12) and (7), we obtain the following symmetrical form of (9)

$\mathrm{d} g_{i j \ldots k}^{\alpha \beta \ldots \gamma} / \mathrm{d} t=\sum_{\mathrm{p}, s \neq i \neq j \ldots \neq k} \gamma_{i s}^{\mathrm{p} \alpha}\left\langle\left(\exp D_{i s}^{\mathrm{p} \alpha}-\exp D_{s i}^{\mathrm{p} \alpha}\right) n_{\mathrm{ri}} n_{\mathrm{rs}} n_{\beta j} \ldots n_{\gamma k}\right\rangle+\{\alpha i \rightarrow \beta j, \ldots \gamma k\}$.

Here

$$
\begin{aligned}
& D_{i s}^{\lambda \alpha}=\beta\left(H_{\lambda i}^{t}+H_{\alpha s}^{t}+H_{\lambda i, \alpha s}^{t}-Q_{\lambda i}^{\prime}-Q_{\alpha s}^{\prime}-Q_{\lambda i, \alpha s}^{\prime}\right) \\
& D_{i s}^{\mathrm{r} \alpha}=\beta\left(H_{\alpha s}^{t}-Q_{\alpha s}^{\prime}\right)+\Psi_{i} \\
& \Psi_{i}=\beta\left(\varphi_{i}^{\mathrm{r}}+\sum_{\nu l} v_{i l}^{\mathrm{r} v} n_{\nu l}\right)
\end{aligned}
$$

where $\varphi_{i}^{\mathrm{r}}=\Phi_{i}^{\mathrm{r}}+\sum_{l} V_{i l}^{\mathrm{rr}}, v_{i l}^{\mathrm{rv}}=V_{i l}^{\mathrm{rv}}-V_{i l}^{\mathrm{rr}}$, and we took into account the $i \rightarrow s$ symmetry of the configuration-independent factor $\gamma_{i s}^{\mathrm{p} \alpha}$ in the jump probability.

For the pairwise interactions under consideration the differences $H_{\alpha s}^{t}-Q_{\alpha s}$ and $H_{\lambda i, \alpha s}^{t}-Q_{\lambda i, \alpha s}^{\prime}$ in (15), according to (2)-(4), can be written as

$$
\begin{aligned}
& H_{\alpha s}^{t}-Q_{\alpha s}=\left(H-Q^{\prime}\right)_{\alpha s}+\varphi_{s}^{\mathrm{r}}+\sum_{\nu l} v_{s l}^{\mathrm{rv}} n_{\nu l} \\
& H_{\lambda i, \alpha s}^{t}-Q_{\lambda i, \alpha s}^{\prime}=\left(H-Q^{\prime}\right)_{\lambda i, \alpha s}+V_{i s}^{\lambda \alpha}-v_{i s}^{\lambda \alpha} .
\end{aligned}
$$

Here $\left(H-Q^{\prime}\right)_{\alpha s}$ means $\left(H_{\alpha s}-Q_{\alpha s}^{\prime}\right)$ where $H_{\alpha s}=\delta H / \delta n_{\alpha s}$ or $Q_{\alpha s}^{\prime}=\delta Q^{\prime} / \delta n_{\alpha s}$ is the variational derivative of the Hamiltonian (3) or the quasi-Hamiltonian (11a), and $\left(H-Q^{\prime}\right)_{\lambda i, \alpha s}$ is the difference of analogous second variational derivatives of $H$ and $Q^{\prime}$ :

$$
\begin{aligned}
& \left(H-Q^{\prime}\right)_{\alpha s}=\lambda_{s}^{\alpha}+\varphi_{s}^{\alpha}+\left(H_{i n t}-Q\right)_{\alpha s} \\
& \left(H-Q^{\prime}\right)_{\lambda i, \alpha s}=\left(H_{i n t}-Q\right)_{\lambda i, \alpha s .}
\end{aligned}
$$

Let us divide the operator $D_{i s}^{\mathrm{p} \alpha}$ in (14) into parts $D_{i s}^{\mathrm{p} \alpha+}$ and $D_{i s}^{\mathrm{p} \alpha-}$, symmetrical and antisymmetrical in indices $i$ and $s: D_{i s}^{\mathrm{p} \alpha}=D_{i s}^{\mathrm{p} \alpha+}+D_{i s}^{\mathrm{p} \alpha-}$. Using (15) and (16), we find

$$
\begin{aligned}
D_{i s}^{\lambda \alpha+}= & \left(\frac{1}{2} \beta\left[\left(H-Q^{\prime}\right)_{\lambda i}+\left(H-Q^{\prime}\right)_{\alpha i}+\left(H-Q^{\prime}\right)_{\lambda i, \alpha s}\right]+\Psi_{i}\right)+\{i \rightarrow s\} \\
\quad+\beta\left(V_{i s}^{\lambda \alpha}-v_{i s}^{\lambda \alpha}\right) & \\
D_{i s}^{\mathrm{r} \alpha+}= & \left(\frac{1}{2} \beta\left(H-Q^{\prime}\right)_{\alpha i}+\Psi_{i}\right)+\{i \rightarrow s\} \\
D_{i s}^{\lambda \alpha-}= & \frac{1}{2} \beta\left[\left(H-Q^{\prime}\right)_{\lambda i}-\left(H-Q^{\prime}\right)_{\alpha i}+\left(H-Q^{\prime}\right)_{\lambda i, \alpha s}\right]-\{i \rightarrow s\} \\
D_{i s}^{\mathrm{r} \alpha-}= & \frac{1}{2} \beta\left[\left(H-Q^{\prime}\right)_{\alpha s}-\left(H-Q^{\prime}\right)_{\alpha i}\right]
\end{aligned}
$$

where we took into account the $i \rightarrow s$ symmetry of the pair potentials $V_{i s}^{\lambda \alpha}$ and $v_{i s}^{\lambda \alpha}$. Then we can write the kinetic equations (14) in the final form that generalizes (14) of [15] to the many-component alloy case:

$$
\begin{aligned}
\mathrm{d} g_{i j \ldots k}^{\alpha \beta \ldots \gamma} / \mathrm{d} t= & \sum_{\mathrm{p}, s \neq i \neq j \ldots \neq k} \gamma_{i s}^{\mathrm{p} \alpha}\left\langle\exp \left(D_{i s}^{\mathrm{p} \alpha+}\right)\left[\exp \left(D_{i s}^{\mathrm{p} \alpha-}\right)-\exp \left(-D_{i s}^{\mathrm{p} \alpha-}\right)\right] n_{\mathrm{ri}} n_{\mathrm{r} s} n_{\beta j} \ldots n_{\gamma k}\right\rangle \\
& +\{\alpha i \rightarrow \beta j, \ldots \gamma k\} .
\end{aligned}
$$

The expression in square brackets in (19) has evidently the meaning of the 'generalized driving force' that determines the evolutionary trends in the atomic distribution, while its 
prefactor plays a role of the generalized mobility. Note that for actual substitution alloys with the vacancy-mediated atomic exchange, the jump probability $\gamma_{i s}^{\mathrm{p} \alpha}$ in (19) is nonzero only when either $\mathrm{p}$ or $\alpha$ species corresponds to the vacancy, i.e. either $\mathrm{p}=\mathrm{v}$ and $\alpha \neq \mathrm{v}$, or $\alpha=\mathrm{v}$ and $\mathrm{p} \neq \mathrm{v}$.

Following the arguments of [15] one can use (19) to prove the ' $H$-theorem', i.e. to define the 'non-equilibrium free energy' having a fundamental property not to increase under spontaneous evolution of the system. To simplify the proof we put the component $r$ in (1) to be a vacancy: $\mathrm{r}=\mathrm{v}$. Then the jump probability $\gamma_{i s}^{\mathrm{p} \alpha}$ in (19) is nonzero only at $\mathrm{p}=\mathrm{r}=\mathrm{v}$, so these equations take the form

$$
\begin{aligned}
\mathrm{d} g_{i j \ldots k}^{\alpha \beta \ldots \gamma} / \mathrm{d} t= & \sum_{s \neq i \neq j \ldots \neq k} \gamma_{i s}^{\mathrm{v} \alpha}\left\langle\exp \left(D_{i s}^{\mathrm{v} \alpha+}\right)\left[\exp \left(D_{i s}^{\mathrm{v} \alpha-}\right)-\exp \left(-D_{i s}^{\mathrm{v} \alpha-}\right)\right] n_{\mathrm{v} i} n_{\mathrm{vs}} n_{\beta j} \ldots n_{\gamma k}\right\rangle \\
& +\{\alpha i \rightarrow \beta j, \ldots \gamma k\} .
\end{aligned}
$$

Let us multiply (20) for $\mathrm{d} g_{i}^{\alpha} / \mathrm{d} t \equiv \mathrm{d} c_{i}^{\alpha} / \mathrm{d} t$ by the factor $\tilde{a}_{i}^{\alpha}=-\left(\lambda_{i}^{\alpha}+\varphi_{i}^{\alpha}\right)$ and sum it over $i$ and $\alpha$; (20) for $\mathrm{d} g_{i_{1} \ldots i_{m}}^{\alpha_{1} \ldots \alpha_{m}} / \mathrm{d} t$ by the factor $\tilde{a}_{1_{1} \ldots i_{m}}^{\alpha_{1} \ldots \alpha_{m}}=a_{i_{1} \ldots i_{m}}^{\alpha_{1} \ldots \alpha_{m}}-v_{i_{1} \ldots i_{m}}^{\alpha_{1} \ldots \alpha_{m}}$ (for the pairwise interaction under consideration the potential $v_{i_{1} \ldots i_{m}}^{\alpha_{1} \ldots 1_{m} \ldots i_{m}}$ at $m \geqslant 3$ is zero) and sum this equation over all $\alpha_{1}, \ldots, \alpha_{m}$ and $i_{1}>\ldots>i_{m}$, etc. Then we sum up all these equations. If we denote for brevity the operator $\exp \left[\beta\left(Q^{\prime}-H\right)_{\alpha i}\right]$ as $\Pi_{\alpha i}$, the resulting relation may be written as

$$
\frac{\mathrm{d} F}{\mathrm{~d} t}=-\frac{1}{2} T \sum_{\alpha, i s}\left\langle A_{i s}^{\mathrm{v} \alpha}\left(\Pi_{\alpha i}-\Pi_{\alpha s}\right) \ln \frac{\Pi_{\alpha i}}{\Pi_{\alpha s}}\right\rangle .
$$

Here a non-negative quantity $A_{i s}^{\mathrm{v} \alpha}$ is expressed via operators $D_{i s}^{\mathrm{v} \alpha+}$ and $\Pi_{\alpha i}$ as

$$
A_{i s}^{\mathrm{v} \alpha}=\gamma_{i s}^{\mathrm{v} \alpha} \exp \left(D_{i s}^{\mathrm{v} \alpha+}\right) n_{\mathrm{v} i} n_{\mathrm{vs}}\left(\Pi_{\alpha i} \Pi_{\alpha s}\right)^{-1 / 2}
$$

while the generalized free energy $F$ is defined by the differential relation analogous to the first law of thermodynamics:

$$
\mathrm{d} F=\sum_{\alpha i}\left(\lambda_{i}^{\alpha}+\varphi_{i}^{\alpha}\right) \mathrm{d} c_{\alpha i}+\sum_{\alpha \ldots \beta, i>\ldots j}\left(v_{i \ldots j}^{\alpha \ldots \beta}-a_{i \ldots j}^{\alpha \ldots \beta}\right) \mathrm{d} g_{i \ldots j}^{\alpha \ldots \beta} .
$$

In the derivation of (21) we took into account that according to $(18 d)$ for $\mathrm{r}=\mathrm{v}$ the operator $\exp \left(D_{i s}^{\mathrm{v} \alpha-}\right)$ is equal to $\left(\Pi_{\alpha s} / \Pi_{\alpha i}\right)^{1 / 2}$, while $\Pi_{\alpha i}$ is related to quantities $\tilde{a}_{i_{1} \ldots i_{m}}^{\alpha_{1} \ldots \alpha_{m}}$ as

$$
\Pi_{\alpha i}=\exp \left[\beta\left(\tilde{a}_{i}^{\alpha}+\sum_{m=2}^{N_{s}} \sum_{\gamma_{2} \ldots \gamma_{m}, j_{2}>\ldots j_{m}} \tilde{a}_{i j_{2} \ldots j_{m}}^{\alpha \gamma_{2} \ldots \gamma_{m}} n_{j_{2}}^{\gamma_{2}} \ldots n_{j_{m}}^{\gamma_{m}}\right)\right]
$$

where $N_{s}$ is the total number of sites in the lattice. As the summand in the right-hand side of (21) is not negative (and is similar in its form to the expression arising in proofs of the $H$-theorem for entropy), the relation (21) shows that the quantity $F$ has the fundamental property not to increase under spontaneous evolution of the system.

To relate the free energy $F$ to the generalized grand canonical potential $\Omega$ we note that according to the definition (11b) the derivatives of the function $\Omega\left\{\lambda_{i}^{\alpha}, a_{i \ldots j}^{\alpha \ldots \beta}\right\}$ over $\lambda_{i}^{\alpha}$ and $a_{i \ldots j}^{\alpha \ldots \beta}$ are equal to $\left(-c_{\alpha i}\right)$ and $g_{i \ldots j}^{\alpha \ldots \beta}$, respectively. Thus the first law of thermodynamics for the potential $\Omega$ has the form

$$
\mathrm{d} \Omega=-\sum_{\alpha i} c_{\alpha i} \mathrm{~d} \lambda_{i}^{\alpha}+\sum_{\alpha \ldots \beta, i>\ldots j} g_{i \ldots j}^{\alpha \ldots \beta} \mathrm{d} a_{i \ldots j}^{\alpha \ldots \beta} .
$$

Comparing (25) and (23) we see that the function $F-\langle H\rangle$ is related to $\Omega$ with the Legendre transformation from variables $a_{i \ldots j}^{\alpha \ldots \beta}$ to $g_{i \ldots j}^{\alpha \ldots \beta}$ :

$$
F-\langle H\rangle=\Omega-\sum_{m=1}^{N_{s}} \sum_{\alpha_{1} \ldots \alpha_{m}, j_{1}>\ldots j_{m}} a_{j_{1} \ldots j_{m}}^{\alpha_{1} \ldots \alpha_{m}} g_{j_{1} \ldots j_{m}}^{\alpha_{1} \ldots \alpha_{m}}
$$


where $a_{i}^{\alpha}$ is $\left(-\lambda_{i}^{\alpha}\right)$. As the last sum in (26) is just the average $\left\langle Q^{\prime}\right\rangle$, the free energy $F$ can also be written as

$$
F=\Omega+\left\langle H-Q^{\prime}\right\rangle
$$

while (23) shows that the derivatives of $F=F\left\{c_{\alpha i}, g_{i \ldots j}^{\alpha \ldots \beta}\right\}$ over its arguments are

$$
\begin{aligned}
& \partial F / \partial c_{\alpha i}=\lambda_{i}^{\alpha}+\varphi_{i}^{\alpha} \\
& \partial F / \partial g_{i \ldots j}^{\alpha \ldots \beta}=v_{i \ldots j}^{\alpha \ldots \beta}-a_{i \ldots j}^{\alpha \ldots \beta} .
\end{aligned}
$$

The above-mentioned $H$-theorem implies that in the stationary state the free energy is minimal with respect to its variables $c_{\alpha i}$ and $g_{i \ldots j}^{\alpha \ldots \beta}$ provided the total number $N_{\alpha}=\sum_{i} c_{\alpha i}$ of atoms of each species is fixed. Then (21) yield the equilibrium conditions having the usual Gibbs-like form:

$$
\begin{aligned}
& \lambda_{i}^{\alpha}+\varphi_{i}^{\alpha}=\mu_{\alpha}=\text { constant } \\
& a_{i \ldots j}^{\alpha \ldots \beta}=v_{i \ldots j}^{\alpha \ldots \beta} \quad \text { or } \quad Q=H_{i n t} .
\end{aligned}
$$

Exact relations of this section can be used for various approximate treatments of the kinetic problems. Such treatments are discussed below.

\section{Approximate expressions for generalized driving forces and mobilities}

To approximately solve (19) one can use various approximate methods of statistical physics, such as the mean-field approximation (MFA), the cluster field method (CFM) [20,21], and the cluster variation method (CVM) [22]. As mentioned in [15], in both MFA and CFM the interaction renormalization effects are neglected, i.e. the 'short-range equilibrium' relations (29b) are supposed to hold. Thus the difference $\left(H_{i n t}-Q\right)$ in (17) vanishes, and (19) yield the closed set of equations for local concentrations $c_{\alpha i}$. Using the relations (17), (18) and (29) we can write these equations as

$$
\frac{\mathrm{d} c_{\alpha i}}{\mathrm{~d} t}=\sum_{\mathrm{p}, s} M_{i s}^{\mathrm{p} \alpha} 2 \sinh \left[\frac{1}{2} \beta\left(F_{\mathrm{p} i}-F_{\mathrm{p} s}-F_{\alpha i}+F_{\alpha s}\right)\right] .
$$

Here $F_{\mathrm{p} i}$ is $\partial F / \partial c_{\mathrm{p} i}$, and for $\mathrm{p}=\mathrm{r}$ the derivative $F_{\mathrm{p} i}$ is zero (similarly to the derivative $Y_{\mathrm{p} i}$ in (13)). The generalized mobility $M_{i s}^{\mathrm{p} \alpha}$ in (30) is determined by the relation

$$
M_{i s}^{\mathrm{p} \alpha}=\gamma_{i s}^{\mathrm{p} \alpha}\left\langle n_{\mathrm{r} i} n_{\mathrm{r} s} \exp \left(D_{i s}^{\mathrm{p} \alpha+}\right)\right\rangle .
$$

In the explicit form, the mobilities can be written as

$$
\begin{aligned}
& M_{i s}^{\lambda \alpha}=\gamma_{i s}^{\lambda \alpha} B_{i s} \exp \left[\beta\left(V_{i s}^{\lambda \alpha}-v_{i s}^{\lambda \alpha}\right)+\frac{1}{2} \beta\left(F_{\lambda i}+F_{\lambda s}+F_{\alpha i}+F_{\alpha s}\right)\right] \\
& M_{i s}^{\mathrm{r} \alpha}=\gamma_{i s}^{\mathrm{r} \alpha} B_{i s} \exp \left[\frac{1}{2} \beta\left(F_{\alpha i}+F_{\alpha s}\right)\right]
\end{aligned}
$$

where the common factor $B_{i s}$ is given by the expression

$B_{i s}=\left\langle n_{\mathrm{ri}} n_{\mathrm{r} s} \mathrm{e}^{\Psi_{i}+\Psi_{s}}\right\rangle=\mathrm{e}^{\beta\left(\varphi_{i}^{\mathrm{r}}+\varphi_{s}^{\mathrm{r}}\right)}\left\langle n_{\mathrm{ri}} n_{\mathrm{r} s} \mathrm{e}^{\beta \sum_{v l}\left(v_{i l}^{\mathrm{rv}}+v_{s l}^{\mathrm{rv}}\right) n_{v l}}\right\rangle$.

At realistically small concentrations of vacancies all terms proportional to $c_{\mathrm{v} i}$ in (33) can be neglected, thus the factor $B_{i s}$ is determined only by the main alloy component distribution.

Equations (30)-(33) are greatly simplified in the MFA. The MFA expressions for the free energy $F\left\{c_{\mathrm{p} i}\right\}$ and its derivatives $F_{\alpha i}$ are

$$
\begin{aligned}
& F=E_{0}+\sum_{\alpha i} \varphi_{i}^{\alpha} c_{\alpha i}+\sum_{\alpha \beta, i>j} v_{i j}^{\alpha \beta} c_{\alpha i} c_{\beta j}+T \sum_{i}\left(c_{\mathrm{ri}} \ln c_{\mathrm{r} i}+\sum_{\alpha} c_{\alpha i} \ln c_{\alpha i}\right) \\
& F_{\alpha i}=\varphi_{i}^{\alpha}+\sum_{\beta j} v_{i j}^{\alpha \beta} c_{\beta j}+T \ln \frac{c_{\alpha i}}{c_{\mathrm{r} i}} .
\end{aligned}
$$


In finding the operator averages the MFA corresponds to replacing each operator $n_{\mathrm{p} i}$ by its mean value $c_{\mathrm{p} i}$ neglecting all possible correlations $[14,13]$. Then (30) take the known MFA form that corresponds to the direct MFA averaging of (9) for $g_{i}^{\alpha}=c_{\alpha i}$, see e.g. [14]:

$\mathrm{d} c_{\alpha i} / \mathrm{d} t=\sum_{\mathrm{p}, s} \gamma_{i s}^{\mathrm{p} \alpha}\left(c_{\mathrm{p} i} c_{\alpha s} \mathrm{e}^{\beta\left[\Phi_{\mathrm{p} i}+\Phi_{\alpha s}+\sum_{\mathrm{q} j}\left(V_{i j}^{\mathrm{pq}}+V_{s j}^{\alpha \mathrm{q}}\right) c_{\mathrm{q} j}\right]}-\{i \rightarrow s\}\right)$.

Note that the factors of the type of $\exp \left(\beta V_{i s}^{\mathrm{pq}}\right)$ in (35) are omitted, in accordance with the MFA validity condition: $\beta V_{i s}^{\mathrm{pq}} \ll 1[13,21]$. For the binary alloy $\mathrm{AB}$ and the direct exchange model (32)-(35) yield the known MFA expression for the mobility [11,23, 15]

$$
M_{i s}^{\mathrm{BA}}=\gamma_{i s}^{\mathrm{BA}}\left\{c_{\mathrm{A} i} c_{\mathrm{A} s} c_{\mathrm{B} i} c_{\mathrm{B} s} \mathrm{e}^{\beta u_{i}+\beta u_{s}}\right\}^{1 / 2}
$$

where

$u_{i}=\Phi_{i}^{\mathrm{A}}+\Phi_{i}^{\mathrm{B}}+\sum_{l}\left(V_{i l}^{\mathrm{AB}}+V_{i l}^{\mathrm{BB}}\right)+\sum_{l}\left(V_{i l}^{\mathrm{AA}}-V_{i l}^{\mathrm{BB}}\right) c_{\mathrm{A} l}=\sum_{\mathrm{p}}\left(\Phi_{i}^{\mathrm{p}}+\sum_{\mathrm{q} l} V_{i l}^{\mathrm{pq}} c_{\mathrm{q} l}\right)$

and indices $\mathrm{p}$ and $\mathrm{q}$ in the last form of (37) take both $\mathrm{A}$ and $\mathrm{B}$ values.

Let us now discuss the treatments of the basic kinetic equation (30) for the $\mathrm{ABv}$ alloy using the CFM. To simplify the notation, below we omit the indices $\mathrm{A}$ and $\mathrm{r}=\mathrm{B}$ when possible, re-label $n_{\mathrm{A} i} \rightarrow n_{i}, c_{\mathrm{A} i} \rightarrow c_{i}$, and also put

$$
\begin{aligned}
& n_{\mathrm{ri}}=n_{\mathrm{B} i} \simeq 1-n_{i} \rightarrow n_{i}^{\prime} \quad c_{\mathrm{B} i} \simeq 1-n_{i} \rightarrow c_{i}^{\prime} \\
& v_{i l}^{\mathrm{AA}}=V_{i l}^{\mathrm{AA}}-2 V_{i l}^{\mathrm{AB}}+V_{i l}^{\mathrm{BB}} \rightarrow v_{i l} .
\end{aligned}
$$

Methods for the CFM calculations of the free energy $F\left\{c_{\alpha i}\right\}$ have been described earlier $[20,21]$ and are simple enough. In particular, in the pair-cluster approximation of CFM (coinciding with that of CVM) the analytical expression for $F\left\{c_{\alpha i}\right\}$ of the $\mathrm{AB}$ alloy was presented in [20] and [21]. Generalizing this approach to the ABv alloy case we obtain the following expressions for the concentration derivatives $F_{\alpha i}$ that enter (30)-(32):

$$
\begin{aligned}
& F_{\mathrm{A} i}=\frac{\partial F}{\partial c_{i}}=\varphi_{i}^{\mathrm{A}}+T \ln \frac{c_{i}}{c_{i}^{\prime}}+T \sum_{j \neq i} \ln \left[1-f_{i j} c_{j} \frac{2}{R_{i j}+1+f_{i j}\left(c_{i}+c_{j}\right)}\right] \\
& F_{\mathrm{v} i}=\frac{\partial F}{\partial c_{\mathrm{v} i}}=\varphi_{i}^{\mathrm{v}}+T \ln \frac{c_{\mathrm{v} i}}{c_{i}^{\prime}}-T \sum_{j \neq i} \ln \left[1+f_{i j}^{\mathrm{v}} c_{j} \frac{2}{R_{i j}+1+f_{i j}\left(c_{i}-c_{j}\right)}\right]
\end{aligned}
$$

Here $f_{i j}=f_{i j}\left(v_{i j}\right)$ or $f_{i j}^{\mathrm{v}}=f_{i j}^{\mathrm{v}}\left(v_{i j}^{\mathrm{vA}}\right)$ is the Mayer function for the potential $v_{i j}$ or $v_{i j}^{\mathrm{vA}}$ :

$$
f_{i j}=\exp \left(-\beta v_{i j}\right)-1 \quad f_{i j}^{\mathrm{v}}=\exp \left(-\beta v_{i j}^{\mathrm{vA}}\right)-1
$$

with $v_{i j}=v_{i j}^{\mathrm{AA}}$ given by (13) and $v_{i j}^{\mathrm{vA}}$ given by (4b) for $\alpha=\mathrm{v}$ and $\beta=\mathrm{A}$ : $v_{i j}^{\mathrm{vA}}=V_{i l}^{\mathrm{vA}}-V_{i l}^{\mathrm{vB}}-V_{i l}^{\mathrm{BA}}+V_{i l}^{\mathrm{BB}}$, while the function $R_{i j}$ is

$$
R_{i j}=\left[1+2 f_{i j}\left(c_{i} c_{j}^{\prime}+c_{i}^{\prime} c_{j}\right)+f_{i j}^{2}\left(c_{i}-c_{j}\right)^{2}\right]^{1 / 2} .
$$

The CFM approach [21] can also be used to calculate the factor $B_{i s}$ (33). The calculations are straightforward but rather cumbersome and will be described elsewhere. For usual interaction models and not too low temperatures, $T \gtrsim 0.5 T_{c}$ where $T_{c}$ is the critical temperature for the alloy decomposition or ordering, the differences between CFM and MFA results for $B_{i s}$ usually do not exceed $10-30 \%$. 


\section{Equivalence of configurational kinetics for the nearest-neighbour vacancy exchange model to that for the direct exchange model}

In this section we show that for the nearest-neighbour vacancy exchange model (being generally accepted and used) the main alloy component kinetics determined by (30) can usually be described in terms of a certain equivalent direct exchange model.

Let us consider a multicomponent $\mathrm{AB}$... Cv alloy with vacancies, e.g. the $\mathrm{ABv}$ alloy, and put $r=B$ in (1), while the other main components $A, \ldots, C$ will be labelled by index $\rho$. Then (30)-(32) can be written as

$$
\begin{aligned}
& \frac{\mathrm{d} c_{\rho i}}{\mathrm{~d} t}=\sum_{s} \tilde{\gamma}_{i s}^{\rho \mathrm{v}} B_{i s}\left[\mathrm{e}^{\beta\left(F_{\rho s}+F_{\mathrm{vi}}\right)}-\mathrm{e}^{\beta\left(F_{\rho i}+F_{\mathrm{vs}}\right)}\right] \\
& \frac{\mathrm{d} c_{\mathrm{v} i}}{\mathrm{~d} t}=\sum_{s} B_{i s}\left[\mathrm{e}^{\beta F_{\mathrm{vs}}}\left(\gamma_{i s}^{\mathrm{vB}}+\sum_{\rho} \tilde{\gamma}_{i s}^{\mathrm{v} \rho} \mathrm{e}^{\beta F_{\rho i}}\right)-\{i \rightarrow s\}\right]
\end{aligned}
$$

where $\tilde{\gamma}_{i s}^{\rho \mathrm{v}}=\gamma_{i s}^{\rho \mathrm{v}} \exp \left[\beta\left(V_{i s}^{\rho \mathrm{v}}-v_{i s}^{\rho \mathrm{v}}\right)\right]$.

The factor $\exp \left(\beta F_{\mathrm{vi}}\right)$ in (42) is proportional to the vacancy concentration $c_{\mathrm{vi}}$, which is illustrated by $(34 b)$ and $(39 b)$ and is actually a general relation of thermodynamics of dilute solutions. Thus the time derivatives of the mean occupations are proportional to the local vacancy concentration $c_{\mathrm{v} i}$ or $c_{\mathrm{v} s}$, which is natural for the vacancy-mediated kinetics. As $c_{\mathrm{v} i}$ is quite small, this implies that the main component relaxation times are by a factor $1 / c_{v i}$ larger than the time of the relaxation of vacancies to their 'quasi-equilibrium' distribution $c_{v i}\left\{c_{\rho i}\right\}$ minimizing the free energy $F\left\{c_{v i}, c_{\rho i}\right\}$ at the given distribution $\left\{c_{\rho i}\right\}$ [24]. Therefore, discarding the small correction of the relative order of $c_{\mathrm{v} i} \ll 1$ we can find this 'adiabatic' vacancy distribution $c_{\mathrm{v} i}$ by equating the left-hand side of $(42 b)$ to zero:

$$
0=\sum_{s} B_{i s} \mathrm{e}^{\beta\left(F_{\mathrm{vi}}+F_{\mathrm{vs}}\right)}\left[\mathrm{e}^{-\beta F_{\mathrm{v} i}}\left(\gamma_{i s}^{\mathrm{vB}}+\sum_{\rho} \tilde{\gamma}_{i s}^{\mathrm{v} \rho} \mathrm{e}^{\beta F_{\rho i}}\right)-\{i \rightarrow s\}\right] .
$$

For the nearest-neighbour vacancy exchange model we can explicitly solve (43) for quantities $\exp \left(\beta F_{\mathrm{vi}}\right)$. In this case the coefficients $\gamma_{i s}^{\mathrm{vB}}=\gamma_{n n}^{\mathrm{vB}}$ and $\tilde{\gamma}_{i s}^{\mathrm{v} \rho}=\tilde{\gamma}_{n n}^{\mathrm{v} \rho}$ are certain constants not depending on indices $i$ and $s$. Then it is convenient to define for each site $i$ a dimensionless quantity $v_{i}$ by the relation

$$
v_{i}=\frac{\gamma_{n n}^{\mathrm{vB}} \exp \left(\beta F_{\mathrm{vi}}\right)}{\left[\gamma_{n n}^{\mathrm{vB}}+\sum_{\rho} \tilde{\gamma}_{n n}^{\mathrm{v} \rho} \exp \left(\beta F_{\rho i}\right)\right] \bar{c}_{\mathrm{v}}}
$$

where $\bar{c}_{\mathrm{v}}$ is the average concentration of vacancies. Then the expression in square brackets (43) is proportional to the difference $v_{i}^{-1}-v_{s}^{-1}$, and the solution of (43) is provided with $v_{i}$ being a constant independent of the site number $i$ (though possibly depending on time, as well as on temperature and other external parameters):

$$
v_{i}=v(t) \text {. }
$$

Relations (44) and (45) determine the above-mentioned adiabatic vacancy distribution $c_{v i}\left\{c_{\rho i}\right\}$. Substituting these relations into (42a) we obtain the kinetic equation for the main alloy component distribution

$$
\frac{\mathrm{d} c_{\rho i}}{\mathrm{~d} t}=\sum_{s} \tilde{\gamma}_{i s}^{\rho \mathrm{v}} B_{i s} \bar{c}_{\mathrm{v}} v(t)\left(\mathrm{e}^{\beta F_{\rho s}}-\mathrm{e}^{\beta F_{\rho i}}\right) .
$$

Comparing (46) with those for the direct exchange model (given by (30)-(32) with $\mathrm{r}=\mathrm{B}$ and $\alpha=\rho$ ) we see that (46) correspond to the direct exchange model with an effective rate

$$
\left(\gamma_{i s}^{\rho \mathrm{B}}\right)_{e f f}=\tilde{\gamma}_{i s}^{\rho \mathrm{v}} \bar{c}_{\mathrm{v}} v(t)
$$


while the direct exchange between the other main components, $\rho$ and $\rho^{\prime}$, is absent: $\left(\gamma_{i s}^{\rho \rho^{\prime}}\right)_{e f f}=0$. Note that the effective direct exchange rate (47) is smaller by a factor $c_{\mathrm{v}}$ than the vacancy exchange rates $\gamma^{\rho \mathrm{v}}$ or $\gamma^{\mathrm{Bv}}$.

Physically, the opportunity to reduce the vacancy-mediated kinetics to the equivalent direct exchange kinetics is connected with the above-mentioned fact that in the course of the alloy evolution the vacancy distribution adiabatically fast follows that of the main components. Therefore, one may suppose that such equivalence holds not only for the approximate kinetic equation (30) and the nearest-neighbour exchange model but is actually a general feature of vacancy-mediated kinetics, though for more general treatments and models the simple algebraic equation (47) can be replaced by some more complex relations.

The function $v(t)$ in (45)-(47) determines the rescaling of time between the actual vacancy-mediated model and the equivalent direct exchange model. Note that for the stationary state the functions $F_{\mathrm{v} i}$ and $F_{\rho i}$ in (44), according to (28a) and (29a), become constant. Thus at 'quasi-stationary' stages of microstructural evolution, in particular, at advanced stages of phase transformation when the new phase precipitates or antiphase domains are well formed, the function $v(t)$ does not depend on time and the rescaling of time becomes linear. Therefore, the asymptotic growth laws and scaling exponents for the direct and the vacancy-mediated exchange mechanisms should be the same. If the adiabaticity condition (45) holds (which can be violated only at very early stages of microstructural evolution, see below), one can study the actual vacancy-mediated dynamics using the equivalent direct exchange model just introducing the 'time rescaling'. For example, for the $\mathrm{ABv}$ alloy with the nearest-neighbour vacancy exchange rate $\tilde{\gamma}_{n n}^{\mathrm{Av}}=\gamma^{\mathrm{Av}}$ the actual time $t$ is related to the reduced time $t_{d e}^{*}=\gamma_{d e} t_{d e}$ of the equivalent direct exchange model by the differential relation

$$
\mathrm{d} t=\mathrm{d} t_{d e}^{*} / v\left(t_{d e}^{*}\right) \gamma^{\mathrm{Av}} \bar{c}_{\mathrm{v}} .
$$

To find $v\left(t_{d e}^{*}\right)$ in such calculations one can assume that at the given main component distribution $\left\{c_{\rho i}\right\}$ the vacancy distribution $c_{\mathrm{v} i}$ is stationary, and thus $v$ can be calculated using in (44) the analytical expressions for such $c_{v i}\left\{c_{\rho i}\right\}$ given in [16], with the normalizing constant expressed via the average vacancy concentration $\bar{c}_{\mathrm{v}}$. The form of the resulting rescaling of time (48) is illustrated in figure 4 below.

Let us make remarks on the region of applicability of basic equations (30) and (42)-(47). These equations describe evolution of alloy states in terms of only local concentrations, i.e. mean occupation numbers $\left\langle n_{\mathrm{p} i}\right\rangle=c_{\mathrm{p} i}$. Such description is complete only when these averages provide a sufficiently full information on the alloy state, i.e. when fluctuations of occupations are physically insignificant. This is evidently the case for the equilibrium homogeneous alloy for which all physical characteristics are obtained by averaging over large volumes including macroscopic numbers of atoms $N \gg 1$, while fluctuative contributions have a relative order of magnitude $N^{-1 / 2}$ or $N^{-1}$ [25]. For a nonequilibrium nonuniform alloy the averages $c_{\mathrm{p} i}$ can provide a sufficiently full description only when the microstructure is somewhat 'coarsened', thus observable quantities correspond to averaging over some sufficiently large volumes (which is the case, e.g., for standard electron microscopy studies). This can imply, for example, that in investigations of phase transformations after a quench of a disordered alloy, the employment of (30) and (42) is useful for description of only long-wave concentrational waves or of microstructures that correspond to the presence of not too small precipitates of a new phase (or antiphase domains) that include a sufficiently large number of atoms. However, just these 'mesoscopic' stages of microstructural evolution appear to attract most interest in both fundamental and applied studies of phase transformations (see e.g. [1-14]). 


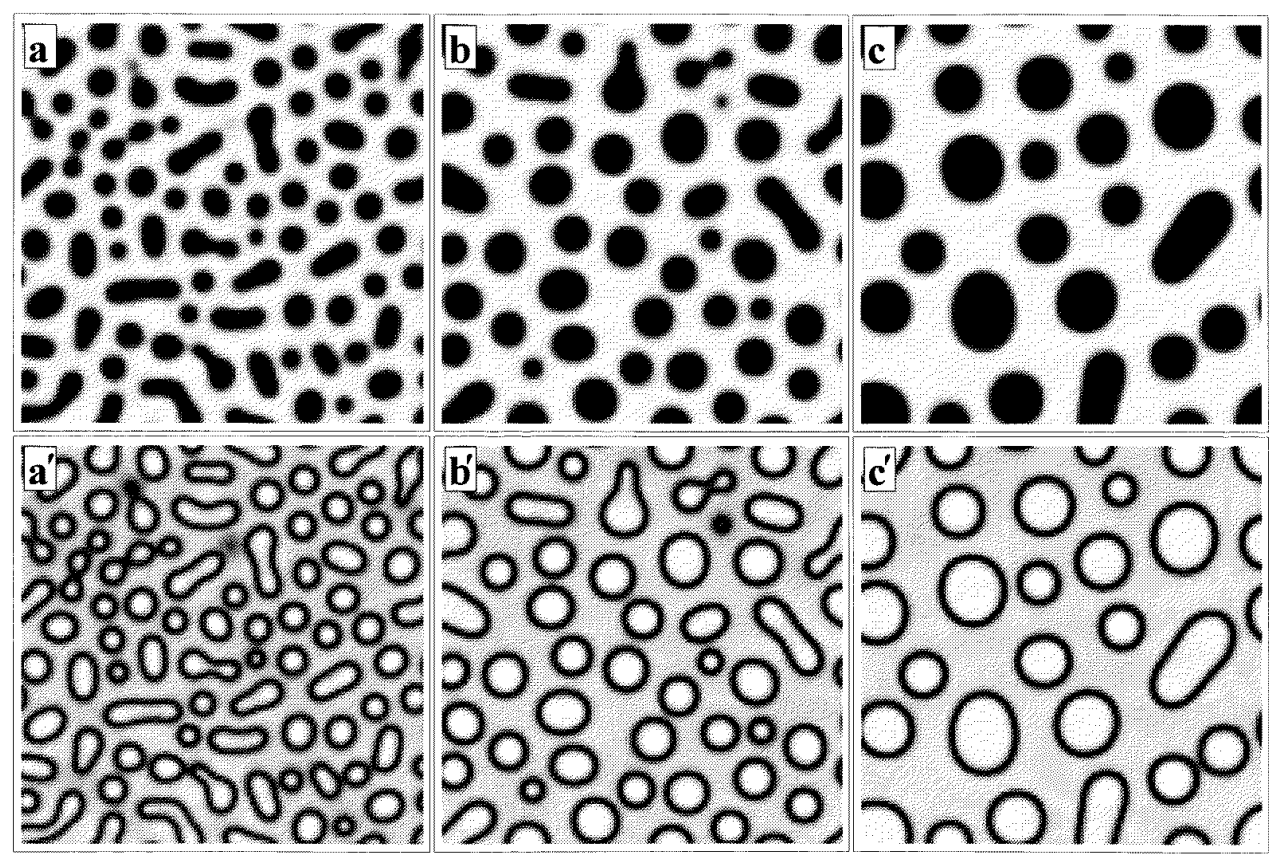

Figure 1. The temporal evolution of mean site occupations, $c_{\mathrm{A} i}=c_{i}$ (upper row), and $c_{\mathrm{v} i}$ (lower row), for the model (i) described in the text at the following values of reduced time

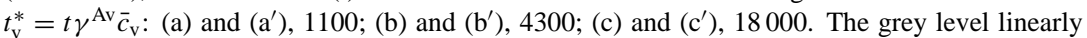
varies with $c_{i}$ or $c_{\mathrm{v} i}$ from completely white to completely black, for $c_{i}$ between $c_{i}=0$ and $c_{i}=1$, and for $c_{\mathrm{v} i}$ between its minimum and maximum value for each of the figure parts; the same colouring is used in figures 2 and 5 below.

For the applicability of the 'adiabatic' relations (43)-(47) the time $t$ after the initial alloy quench into the thermodynamic instability region should exceed the effective time $\tau_{\mathrm{v} d}$ for the vacancy diffusion over a mean inter-vacancy distance $l_{\mathrm{vv}}$. At lesser times, i.e. at very early stages of phase transformations, fluctuations in the initial vacancy distribution can significantly affect the alloy evolution. This is discussed in the next section.

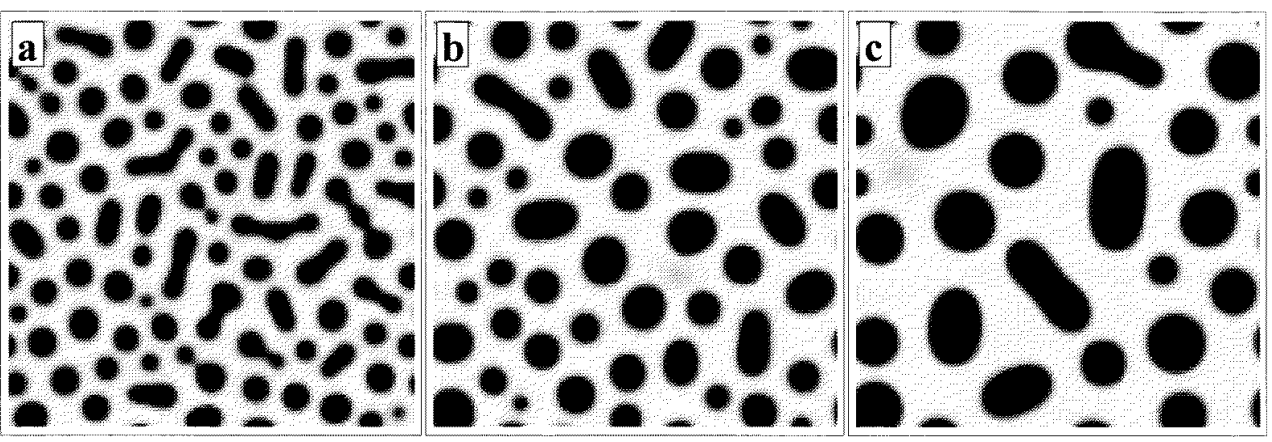

Figure 2. Temporal evolution of $c_{i}$ for model (i) via the direct exchange mechanism at the following values of reduced time $t_{d e}^{*}=t \gamma_{d e}$ : (a) 230, (b) 1070 and (c) 5200 . 


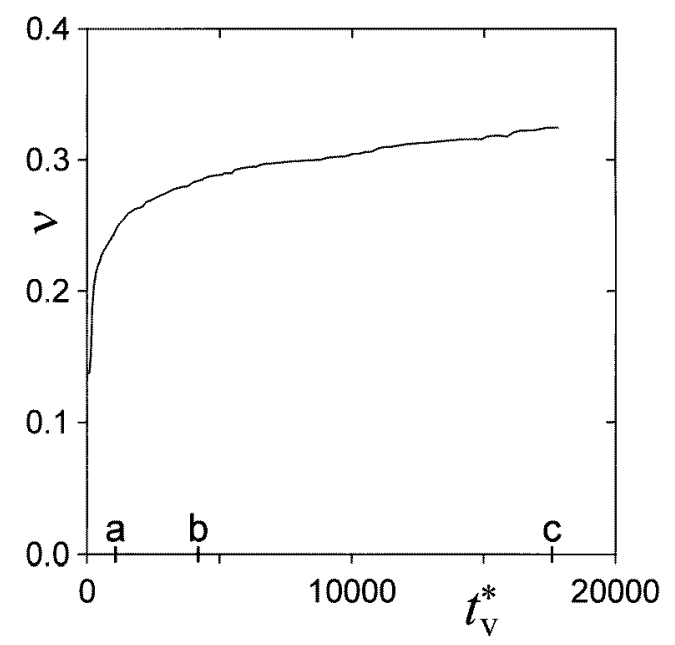

Figure 3. The time dependence of the function $v(t)$ (45) for model (i).

\section{Computer simulation of phase transformations with vacancy-mediated dynamics}

To illustrate the results of the previous section and to obtain an idea of the form of the function $v(t)$ in (44)-(47) we performed computer simulation of phase transformations for two-dimensional $\mathrm{ABv}$ alloy models using the simplest MFA (35). We consider the square lattice containing $128 \times 128$ sites with periodic boundary conditions and interactions in three coordination spheres: $v_{1}, v_{2}$ and $v_{3}$. Two models have been studied: (i) $v_{1}=-1$, $v_{2}=-0.8, v_{3}=-0.5, c_{0}=0.35$ and $T=0.5 T_{c}\left(c_{0}\right.$ and $T_{c}$ being the average alloy concentration and the critical temperature, respectively), which corresponds to the phaseseparating alloy, and (ii) that studied in [17] with $v_{1}=1, v_{2}=-0.8, v_{3}=-0.5, c_{0}=0.325$ and $T=0.424 T_{c}$, which corresponds to the alloy undergoing phase separation with ordering. For simplicity, vacancies are supposed not to interact with both alloy atoms and each other: $V_{i j}^{\mathrm{Av}}=V_{i j}^{\mathrm{Bv}}=V_{i j}^{\mathrm{vv}}=0$, while the external fields $\varphi_{i}(4 a)$ and the asymmetrical potentials $V_{i j}^{\mathrm{AA}}-V_{i j}^{\mathrm{BB}}$ are zero. The $\mathrm{A}-\mathrm{v}$ and $\mathrm{B}-\mathrm{v}$ exchange rates are nonzero only for the nearestneighbours and are supposed to be related as $\gamma_{n n}^{\mathrm{Bv}}=\gamma^{\mathrm{Av}} \exp \left(\beta v_{0} / 2\right)$ where $v_{0}=\sum_{j} v_{i j}$ (this relation makes the function $v$ to be symmetric with respect to both alloy components, A and B). For both models the initial state corresponds to the uniform disordered alloy quenched to the temperature $T$, with the average A component concentration $\left\langle c_{i}\right\rangle=c_{0}$, and its random fluctuations $\delta c_{i}= \pm 0.01$. The first and the second models contain 160 and 16 randomly distributed vacancies (i.e. $\bar{c}_{\mathrm{v}} \simeq 10^{-2}$ and $10^{-3}$ ), respectively.

The results of the simulations are presented in figures $1-7$. Points $\mathrm{a}, \mathrm{b}$ and $\mathrm{c}$ in figures 3 and 4 correspond to the states shown in figures 1 and 2, while points a, b, c and d in figure 6 correspond to the states shown in figure 5 .

Let us first discuss figures 1-4 that describe the phase separation. Significant changes of microstructure for this case correspond to the formation and evolution of the new phase precipitates that include a large number of atoms. Such changes are realized via diffusion of atoms over large distances, and thus they make many atomic jumps. As figures 1-4 describe just such slow changes, values of the reduced time $t_{\mathrm{v}}^{*}=t \gamma^{\mathrm{Av}} \bar{c}_{\mathrm{v}}$ for them are large and much exceed the time $\tau_{\mathrm{v} d}^{*}=\tau_{\mathrm{v} d} \gamma^{\mathrm{Av}} \bar{c}_{\mathrm{v}} \sim 1$ needed for the vacancy distribution equilibration mentioned in section 4 and discussed below. Therefore, effects of fluctuations 
in the initial vacancy distribution for the decomposition case are unimportant, unlike the alloy ordering case considered below. In particular, fluctuations of quantities $v_{i}(44)$ around their average value $\left\langle v_{i}\right\rangle=v(t)$ at $t_{\mathrm{v}}^{*}>\tau_{\mathrm{v} d}^{*}$ become small (similarly to those shown in figure 6 for the ordering case), and for the states shown in figure 1 their magnitude is less than one per cent. Therefore, the microstructural evolution for $t_{\mathrm{v}}^{*} \gg \tau_{\mathrm{v} d}^{*}$ can be described in terms of the direct exchange model (47).

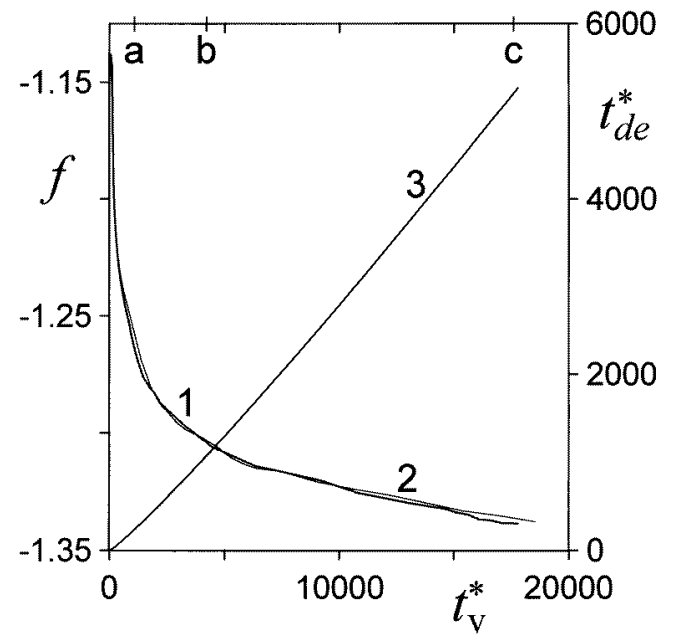

Figure 4. Curve 1 (left scale): time dependence of the reduced free energy per site $f=\beta F / N_{s}$ for model (i) and the vacancy exchange mechanism. Curve 2 (left scale): the same for the direct exchange mechanism. Curve 3 (right scale): time dependence of the reduced direct exchange time $t_{d e}^{*}\left(t_{\mathrm{v}}^{*}\right)$.

Comparison of figure 1(a)-(c) with figure 2 confirms this conclusion: all features of microstructural evolution, such as the distribution of sizes, shapes and density of precipitates, for the vacancy and direct exchange models are similar. Figure $1\left(a^{\prime}\right)-\left(c^{\prime}\right)$ also illustrates a significant segregation of vacancies at interphase boundaries, in accordance with the results of [16] for the model under consideration. Figure 1 also shows a notable enhancement of vacancies in domains of sharp changes of local microstructure (such as vicinities of 'evaporating' precipitates in figure $1\left(\mathrm{a}^{\prime}\right)$ and $\left(\mathrm{b}^{\prime}\right)$ ), or coagulating precipitates in figure $1\left(\mathrm{a}^{\prime}\right)$, which is a characteristic feature of vacancy-mediated kinetics.

Figure 3 shows the time dependence of the function $v(t)$ that is due to the evolution of the main alloy component distribution. The asymptotic value of this function (given by (44) with the equilibrium values of $F_{\mathrm{v} i}$ and $F_{\mathrm{A} i}$ ) is $v(\infty) \simeq 0.51$. Figure 4 shows the reduced free energy per site $f\left(t_{\mathrm{v}}^{*}\right)=\beta F / N_{s}$ (where $N_{s}$ is the same as in (24)), and the same quantity for the equivalent direct exchange model, $f_{d e}\left(t_{\mathrm{v}}^{*}\right)=f\left[t_{d e}^{*}\left(t_{\mathrm{v}}^{*}\right)\right]$, where the dependence $t_{d e}^{*}\left(t_{\mathrm{v}}^{*}\right)$ (curve 3 in figure 4 ) is determined by (48). It is seen that the time dependence of free energy for both kinetic models is virtually the same.

The equivalence of the vacancy-mediated and the direct exchange kinetics at not small $t_{\mathrm{v}}^{*} \gg \tau_{\mathrm{v} d}^{*}$ is also confirmed with simulations of alloy ordering for model (ii), (see figures 5 and 6). In particular, the microstructure in figure 5(d) is similar to that for the direct exchange model shown in figure 5(a) of [17], and, according to (45)-(47), an analogous similarity should hold for later times. Figures $5\left(a^{\prime}\right)-\left(d^{\prime}\right)$ and $7(c)$ illustrate the vacancy ordering accompanying the main component ordering which for the equilibrium case was 

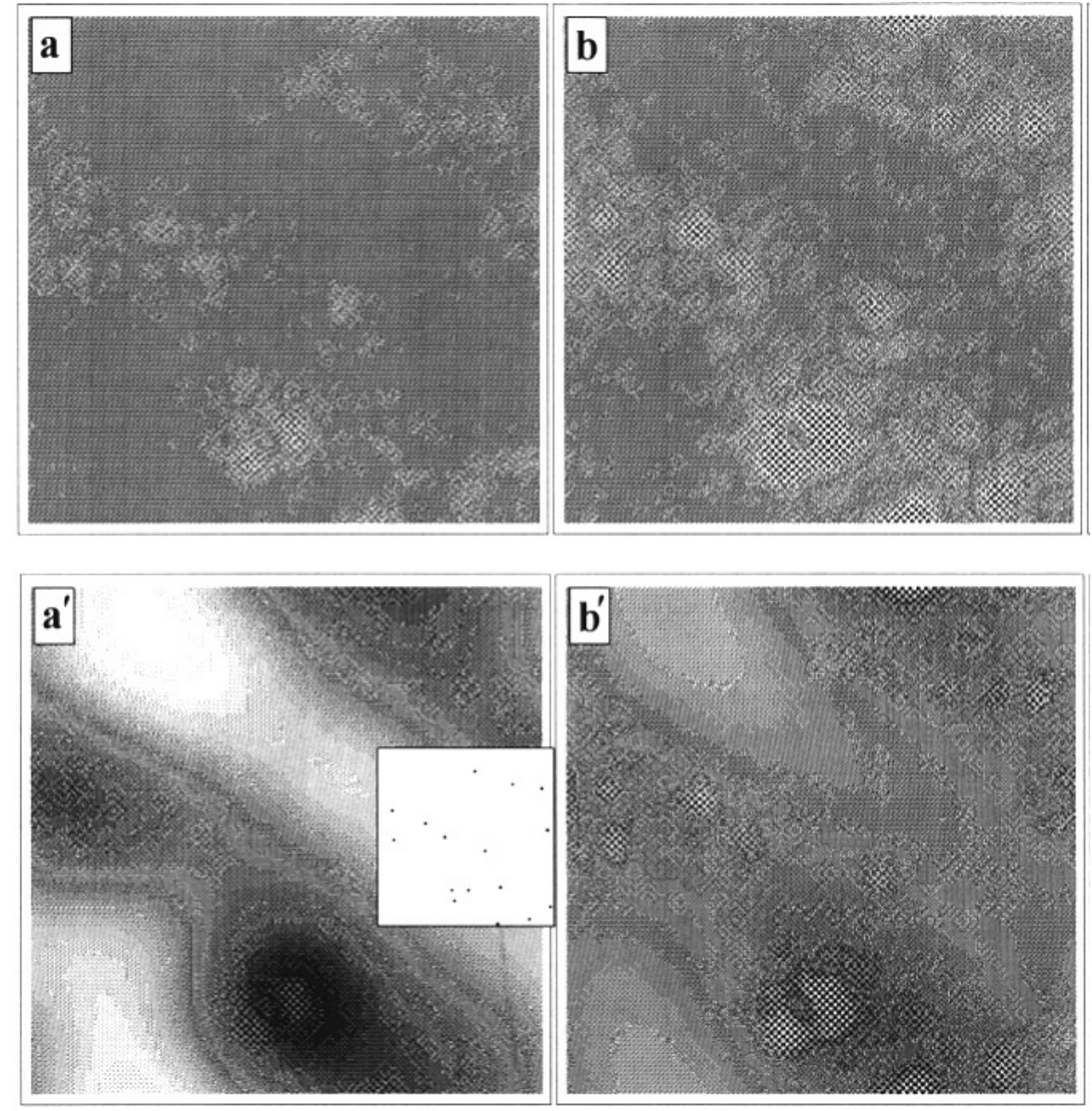

Figure 5. The same as in figure 1 but for model (ii) described in text at the following values of reduced time $t_{\mathrm{v}}^{*}$ : (a) and $\left(\mathrm{a}^{\prime}\right), 0.24$; (b) and $\left(\mathrm{b}^{\prime}\right), 0.49$; (c) and $\left(\mathrm{c}^{\prime}\right), 1.27$; (d) and $\left(\mathrm{d}^{\prime}\right), 4.05$. The inset in $1\left(\mathrm{a}^{\prime}\right)$ shows the initial distribution of vacancies at $t_{\mathrm{v}}^{*}=0$.

discussed in [16] and was described with (31) and (32) of that work. Figure 5(c') and $\left(\mathrm{d}^{\prime}\right)$ also shows a notable segregation of vacancies at antiphase boundaries, which again agrees with the results of [16].

In the rest of this section we discuss features of the vacancy-mediated kinetics at very early stages of ordering, $t \lesssim \tau_{\mathrm{v} d}$. The features are related to fluctuations in the initial vacancy distribution and are illustrated in figures 5(a)-(c), 6 and 7. Let us first discuss the characteristic relaxation times. To this end we consider the time dependence of the value $v(t)=\left\langle v_{i}\right\rangle$ averaged over the lattice, its mean square deviation $\Delta v(t)=\left\langle\left(v_{i}-v\right)^{2}\right\rangle^{1 / 2}$, and the reduced free energy $f(t)=\beta F / N_{s}$ shown in figure 6. It is seen that the deviation $\Delta v$ is initially quite large which is due to the vacancy localization in the initial as-quenched state (shown as an inset in figure $5\left(\mathrm{a}^{\prime}\right)$ ). However, the subsequent diffusion of vacancies results in a sharp decrease of fluctuations $\Delta v_{i}$, and after a certain time $\tau_{\mathrm{v} d}$ they become quite small. The value $\tau_{\mathrm{v} d}$ can be estimated as the time needed for vacancies to diffuse over the whole lattice, i.e to visit each lattice site approximately once. It implies the relation $a^{2} \gamma \tau_{\mathrm{v} d} \sim l_{\mathrm{vv}}^{2}$, where $a$ is the lattice constant, $\gamma$ is of the order of $\gamma^{\mathrm{Av}}$ or $\gamma^{\mathrm{Bv}}$, and $l_{\mathrm{vv}}$ is the 

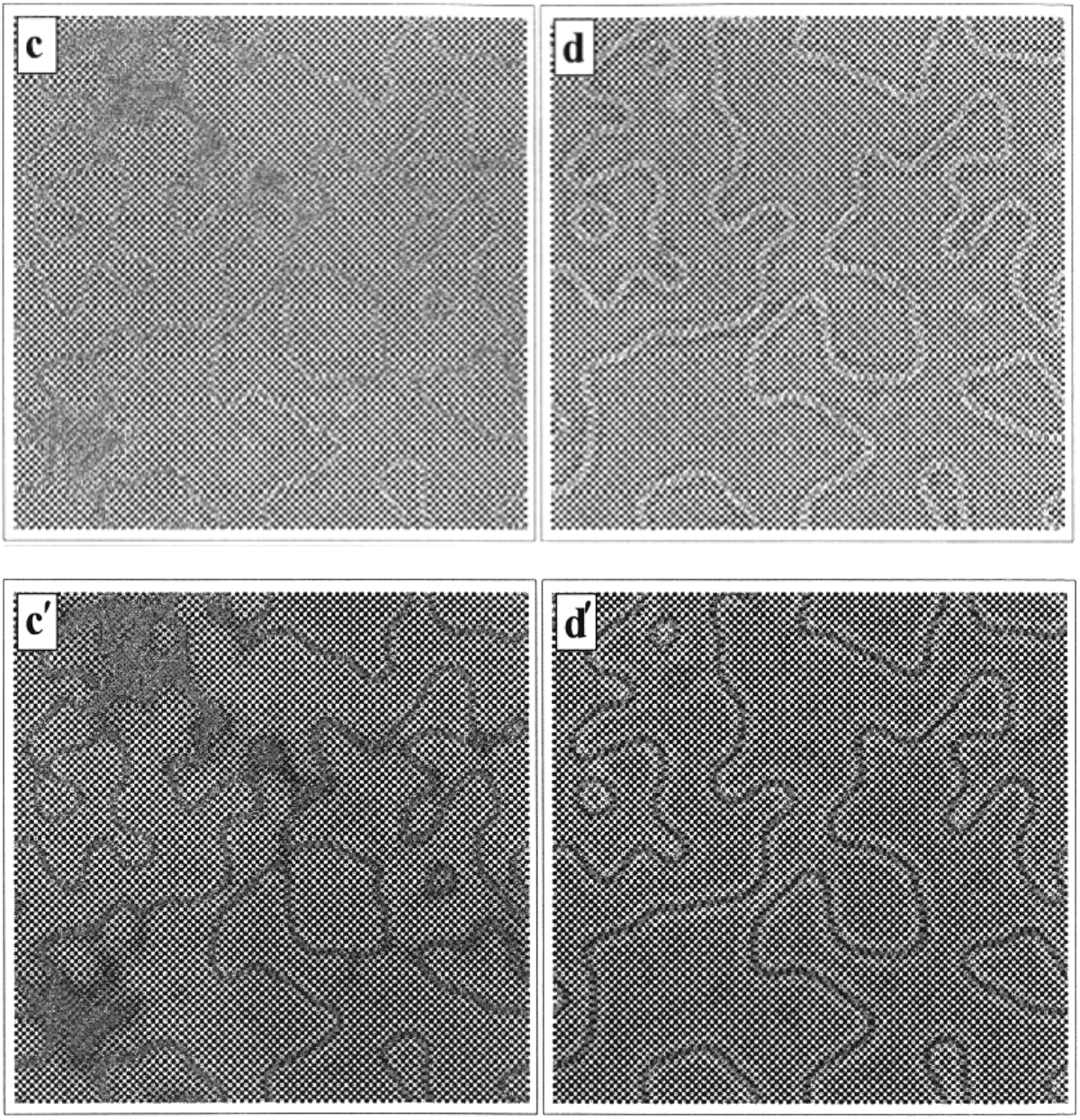

Figure 5. (Continued)

average inter-vacancy distance. For a $d$-dimensional lattice, $l_{\mathrm{vv}}$ is of the order of $a\left(\bar{c}_{\mathrm{v}}\right)^{-1 / d}$ which gives

$$
\tau_{\mathrm{v} d} \sim 1 / \gamma\left(\bar{c}_{\mathrm{v}}\right)^{2 / d} .
$$

For a 2D alloy the estimate yields $\tau_{\mathrm{v} d}^{*} \sim 1$, which agrees with the order of magnitude of the relaxation time for the deviation $\Delta v(t)$ in figure 6 .

At the same time, the characteristic relaxation time for ordering is determined by the time $\tau_{\mathrm{AB}}$ needed for one effective exchange of neighbouring $\mathrm{A}$ and $\mathrm{B}$ atoms. This time can be estimated from the relation $t_{d e}^{*}\left(\tau_{\mathrm{AB}}\right) \sim 1$, which according to (48) gives

$$
\tau_{\mathrm{AB}} \sim 1 / \gamma^{\mathrm{Av}} \nu \bar{c}_{\mathrm{v}} .
$$

Comparison of estimates (49) and (50) shows that two relaxation times are related as $\tau_{\mathrm{v} d} \sim \tau_{\mathrm{AB}} v\left(\bar{c}_{\mathrm{v}}\right)^{1-2 / d}$. For 3D alloys it means that $\tau_{\mathrm{v} d}$ is, generally, by a factor $\left(\bar{c}_{\mathrm{v}}\right)^{1 / 3}$ smaller than $\tau_{\mathrm{AB}}$. For a 2D alloy and $v \sim 1$ (which corresponds to model (ii) under consideration) the estimate yields $\tau_{\mathrm{v} d} \sim \tau_{\mathrm{AB}}$. 


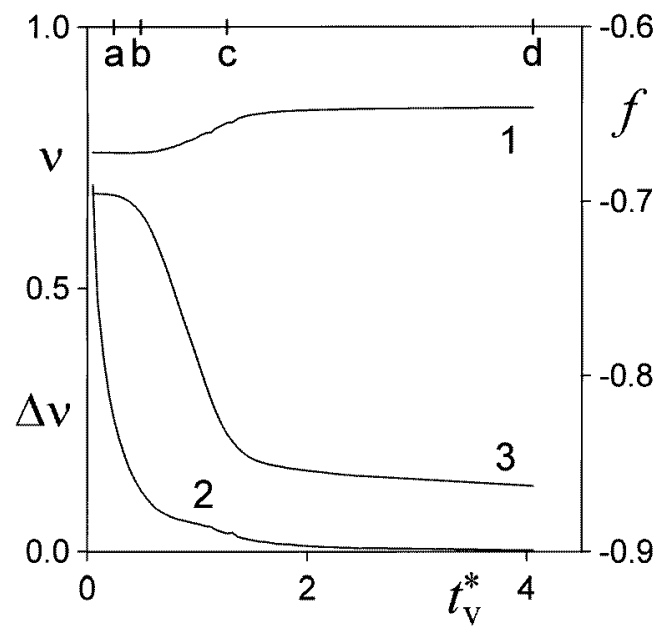

Figure 6. The time dependence of the value $v(t)=\left\langle v_{i}\right\rangle$ averaged over the lattice (curve 1, left scale), its mean square deviation $\Delta v=\left\langle\left(v_{i}-v\right)^{2}\right\rangle^{1 / 2}$ (curve 2, left scale) and the reduced free energy $f(t)=\beta F / N_{s}$ (curve 3, right scale) for model (ii).

To discuss the time dependence of functions $v(t)$ and $f(t)$ in figure 6 it is convenient to define the local mean composition $\bar{c}_{i}$ and the local order parameter $\eta_{i}$ [16]:

$$
\bar{c}_{i}=\frac{1}{2}\left(c_{i}+\frac{1}{4} \sum_{j=n n(i)} c_{j}\right) \quad \eta_{i}=\frac{1}{2}\left(c_{i}-\frac{1}{4} \sum_{j=n n(i)} c_{j}\right) \exp \left(\mathrm{i} \boldsymbol{k}_{s} \boldsymbol{r}_{i}\right)
$$

where index $n n(i)$ means summation over nearest-neighbours of site $i, \boldsymbol{k}_{s}=(1,1) 2 \pi / a$ is the superstructure vector and $\boldsymbol{r}_{i}=\left(x_{i}, y_{i}\right)$ is the lattice site vector. At smallest $t \ll \tau_{\mathrm{AB}}$ the local order parameters $\eta_{i}$ grow with $t$ linearly. As both $v$ and $f$ cannot depend on the sign of the order parameter, they should depend on $\eta_{i}$ and $t$ at small $t$ quadratically. The subsequent ordering results in some increase of $v(t)$ and the decrease of $f(t)$. The time $t \sim \tau_{\mathrm{AB}}$ corresponds to an approximate completion of the initial congruent ordering that occurs at approximately constant local composition $\bar{c}_{i} \simeq c_{0}[17,19]$. The subsequent alloy decomposition into the ordered and disordered phase needs much longer times $t \gg \tau_{\mathrm{AB}}$, see [17] and figures 1-4, thus the relevant variations of functions $v\left(t_{\mathrm{v}}^{*}\right)$ and $f\left(t_{\mathrm{v}}^{*}\right)$ in figure 6 become very slow.

Microstructural evolution at early stages of ordering, $t \lesssim \tau_{v d}$, is illustrated in figure 5(a) and (b). Its prominent feature is the presence of localized (or heterogeneous) ordering, i.e. formation and growth of isolated ordered domains within the disordered medium. As alloy states under consideration are thermodynamically unstable with respect to ordering (their composition $c_{0}$ and temperature $T$ correspond to the region below the ordering spinodal in the $c_{0}, T$ plane [17]), the localized ordering is a characteristic feature of the vacancymediated kinetics that is absent for the direct exchange model.

The phenomenon of localized ordering was first observed by Allen and Cahn (AC) in their study of B2 ordering in $\mathrm{Fe}-\mathrm{Al}$ alloys [19]. To interpret it, AC suggested that the ordering of each domain was generated by a single vacancy during the initial alloy quench from the high-temperature disordered phase. The vacancy was supposed to be trapped at the interface between ordered and disordered materials, in an analogy with a similar trapping observed in some computer simulations for equilibrium interfaces. These suggestions led $\mathrm{AC}$ to the conclusion that there should be an approximate one-to-one correspondence 

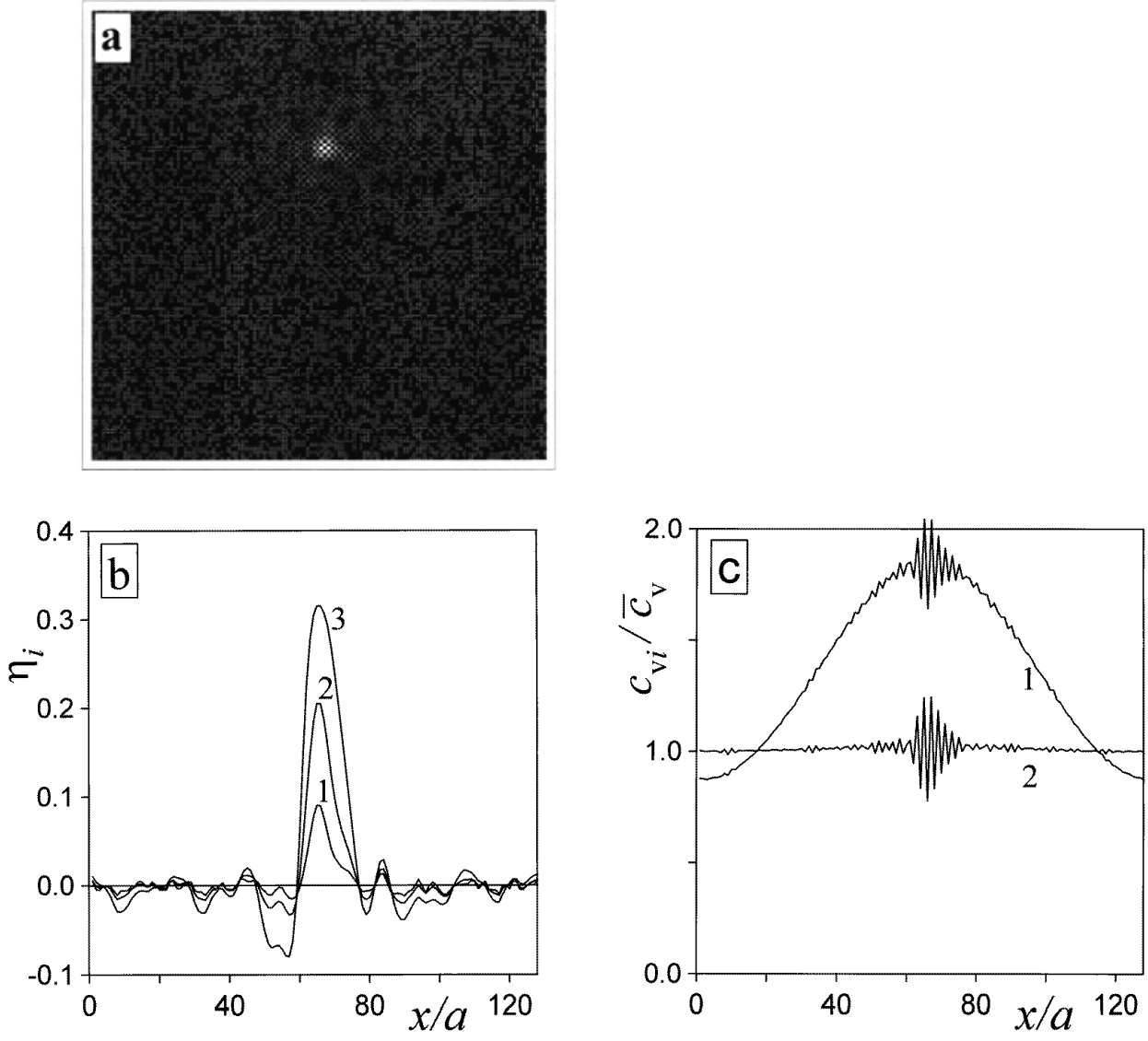

Figure 7. The temporal evolution of model (ii) with a single vacancy in the $128 \times 128$ lattice (a) Local concentrations $c_{i}$ at the dimensionless time value $t^{\prime}=t \gamma^{\mathrm{Av}}=1000$; the grey level linearly varies with $c_{i}$ between its minimum, $c_{\min } \simeq 0.23$, and maximum, $c_{\max } \simeq 0.43$. (b) The local order parameter $\eta_{i}=\eta\left(x_{i}\right)$ along the line $y_{i}=87 a$ in (a); for curves 1,2 and $3, t^{\prime}$ is 1000 , 3000 and 7000 , respectively. (c) The same as in (b) but for the local vacancy concentration $c_{\mathrm{v} i}=c_{\mathrm{v}}\left(x_{i}\right)$; for curves 1 and $2, t^{\prime}$ is 1000 and 3000 .

between the initial vacancy number $N_{\mathrm{v}}=N_{s} \bar{c}_{\mathrm{v}}$ and the observed ordered domain number $N_{o d}=N_{s} c_{o d}$.

In more detail the localized ordering phenomenon was recently discussed by Athenes et al [7]. These authors made a Monte Carlo simulation of B2 ordering in BCC alloys with a single vacancy in the large simulation box. For certain values of the kinetic parameters (being similar to those used in our model (ii)) Athenes et al observed the localized ordering and concluded that this observation confirms the above-mentioned suggestions of AC [19]. Athenes et al also made numerical estimates of concentrations $\bar{c}_{\mathrm{v}}$ and $c_{\text {od }}$ for AC experiments and found $\left(\bar{c}_{\mathrm{v}}\right)_{\text {exp }} \simeq(2-40) \times 10^{-4} ;\left(c_{\text {od }}\right)_{\text {exp }} \simeq 5 \times 10^{-6}$. Let us note that these estimates (even with their uncertainty) seem to suggest an inequality $\left(\bar{c}_{\mathrm{v}}\right)_{\text {exp }} \gg\left(c_{\text {od }}\right)_{\text {exp }}$ rather than the similarity $\left(\bar{c}_{\mathrm{v}}\right)_{\text {exp }} \simeq\left(c_{o d}\right)_{\text {exp }}$ supposed by AC.

The results of our simulation illustrated by figure 5(a) and (b) agree with the general conclusions of $\mathrm{AC}$ and Athenes et al that the localized ordering phenomenon is due to the 
inhomogeneity of the initial vacancy distribution. However, our figure $5\left(\mathrm{a}^{\prime}\right)$ and $\left(\mathrm{b}^{\prime}\right)$ (as well as 7(c)) do not reveal any vacancy segregation (or 'trapping') at domain boundaries for these highly nonequilibrium states, unlike the case of later, partly equilibrated states shown in figure $5\left(\mathrm{c}^{\prime}\right)$ and $\left(\mathrm{d}^{\prime}\right)$, or fully equilibrium boundaries studied in [16]. At the same time, the results of these our simulations seem to imply that the localized ordering can be related, not to the vacancy trapping, but to the vacancy concentration fluctuations which result in the localization of the initial ordering in the regions enriched by vacancies (see figure 5). Let us also note that the mean inter-vacancy distance in our simulation, $l_{\mathrm{vv}} \sim a\left(\bar{c}_{\mathrm{v}}\right)^{-1 / 2} \simeq 30 a$, seems to be of the same order of magnitude as that in AC experiments, $\left(l_{\mathrm{vv}}\right)_{\text {exp }} \sim a\left(\bar{c}_{\mathrm{v}}\right)_{\text {exp }}^{-1 / 3}$, thus the inter-vacancy distance fluctuations may have a similar scale, too. This 'fluctuative' interpretation of localized ordering can also explain the above-mentioned inequality $\left(\bar{c}_{\mathrm{v}}\right)_{\text {exp }} \gg\left(\bar{c}_{\text {od }}\right)_{\text {exp }}$ : the total number of such vacancy-enriched regions is evidently much lower than the total vacancy number.

To make a more direct comparison of our results with those of the Monte Carlo simulation [7], we also simulated ordering in our model (ii) realized by a single vacancy in the simulation box of $128 \times 128$ sites. Our results presented in figure 7 are qualitatively similar to those of Athenes et al [7]. In particular, figure 7(a) and (b) shows the presence of localized ordering. However, figure 7(c) again illustrates the absence of any interfacial vacancy trapping. As discussed in [16], such trapping is characteristic of the equilibrium interfaces and is due to the relevant thermodynamic gain, while in the non-equilibrium states under consideration there seems to be no significant driving force for such trapping.

\section{Conclusion}

Let us summarize the main results of this work. To theoretically describe the evolution of atomic distributions in non-equilibrium alloys via the realistic vacancy exchange mechanism we generalize the earlier-suggested master equation approach to the many-component alloy case. We present exact kinetic equations for average occupations of lattice sites and various correlators of these occupations. We derive the expression for free energy of a non-equilibrium alloy that has a fundamental property to not increase under spontaneous evolution of the system. We also present approximate forms of kinetic equations which correspond to mean-field and cluster field approximations.

These equations are used to show that, for the nearest-neighbour vacancy exchange model, the evolution of the main alloy component distribution $\left\{c_{\rho i}\right\}$ can usually be described in terms of an equivalent direct exchange model with some effective exchange rates that, generally, depend on time. Physically, the equivalence is due to the fact that the relaxation of distribution $\left\{c_{\rho i}\right\}$ via vacancy exchange at small vacancy concentration $\bar{c}_{\mathrm{v}}$ occurs more slowly by a factor $1 / \bar{c}_{\mathrm{v}}$ than that of the vacancy distribution. Therefore, the distribution of vacancies adiabatically fast follows that of main components and corresponds to their conditional equilibrium at the given $\left\{c_{\rho i}\right\}$. These 'adiabatic' conditions can be violated only under a sharp change of external parameters, such as a rapid alloy quench into the region of its instability with respect to phase transformations. Then some 'non-adiabatic' effects can occur at initial stages of evolution.

These conclusions are illustrated by simulations of decomposition and ordering via vacancy-mediated exchange for a two-dimensional model of a binary alloy, with the use of kinetic equations corresponding to the MFA. It is shown that for the decomposition case the mentioned non-adiabatic effects are insignificant, and all features of microstructural evolution do coincide with those for the equivalent direct exchange model. The last 
conclusion is also found to be true for the ordering case, except only for very initial stages of ordering.

At these initial stages, a peculiar phenomenon of localized ordering has been observed in our simulations, in agreement with the experiments [19] and Monte Carlo simulations [7]. However, contrary to suggestions made in previous works, no vacancy trapping at the ordered domain boundaries has been found for this initial period, and effects of spatial fluctuations in the initial vacancy distribution seem to provide a more adequate explanation for the localized ordering phenomenon.

\section{Acknowledgments}

The authors are much indebted to Georges Martin for numerous illuminating discussions. The work was supported by the Russian Fund of Basic Research, grant No 97-02-17842.

\section{References}

[1] Yaldram K and Binder K 1991 Acta Metall. 39707 Yaldram K and Binder K 1991 J. Stat. Phys. 62161

[2] Yaldram K and Binder K 1991 Z. Phys. B 82405

[3] Fratzl P and Penrose O 1994 Phys. Rev. B 503477

[4] Vives E and Planes A 1993 Phys. Rev. B 472557

[5] Frontera C, Vives E and Planes A 1994 Z. Phys. B 9679

[6] Soisson F, Barbu A and Martin G 1996 Acta Mater. 443789

[7] Athenes M, Bellon P, Martin G and Haider F 1996 Acta Mater. 444739

[8] Geng C and Chen L Q 1994 Scripta Metall. Mater. 311507

[9] Chen L Q 1994 Mater. Res. Soc. Symp. Proc. vol 319 (Pittsburgh, PA: Material Research Society) p 375

[10] Amar J C, Sullivan F E and Mountain R D 1988 Phys. Rev. B 37196

[11] Martin G 1990 Phys. Rev. B 412279

[12] Chen L Q, Wang Y Z and Khachaturyan A G 1994 Statics and Dynamics of Alloy Phase Transformations (NATO Advanced Study Institute, Series B: Physics 319) ed A Gonis and P E A Turchi (New York: Plenum) p 587

[13] Vaks V G and Beiden S V 1994 Zh. Eksp. Teor. Fiz. 1051017 (Engl. Transl. Sov. Phys.-JETP 78 546)

[14] Vaks V G, Beiden S V and Dobretsov V Yu 1995 Pis. Zh. Eksp.Teor. Fiz. 6165 (Engl. Transl. JETP Lett. $6168)$

[15] Vaks V G 1996 Pis. Zh. Eksp.Teor. Fiz. 63447 (Engl. Transl. JETP Lett. 63 471)

[16] Belashchenko K D and Vaks V G 1997 Zh. Eksp. Teor. Fiz. 112714

[17] Dobretsov V Yu, Vaks V G and Martin G 1996 Phys. Rev. B 543227

[18] Chen L Q 1994 Phys. Rev. B 493791

[19] Allen S M and Cahn J W 1976 Acta Metall. 24425

[20] Vaks V G and Orlov V G 1986 Fiz. Tverd. Tela 283627

[21] Vaks V G, Zein N E and Kamyshenko V V 1988 J. Phys. F: Met. Phys. 181641

[22] Sanchez J M, Ducastelle F and Gratias D 1984 Physica A 128334

[23] Martin G 1994 Phys. Rev. B 5012362

[24] Abromeit C 1989 Int. J. Mod. Phys. B 31301

[25] Landau L D and Lifshits E M 1976 Statistical Physics (Moscow: Nauka) section 2 